\title{
$\therefore$ Interaction of Correction of Lipid Peroxidation Disorders with Oxibral
}

\author{
IJCRR \\ Section: Healthcare \\ ISI Impact Factor \\ (2019-20): 1.628 \\ IC Value (2019): 90.81 \\ SJIF $(2020)=7.893$ \\ (c) (7) (8) \\ Copyright@IJCRR
}

Sharipov R. Kh., Akhmedova M.M., Rasulova N.A.,, Erbutayeva L.T.

Samarkand State Medical lnstitute, PhD., Teacher of the Department of Pediatrics FADE, Samarkand, Uzbekistan.

\section{ABSTRACT}

Introduction: Cerebral pathology is leading reason of not only morbidity and death rate but also heavy disability in subsequent ontogenesis At the of aggressive free radicals, hydroperoxides that render the destructive operating on the membranes of neurons.

Objective: To ground the efficiency of therapy of "oxibral" of pectoral children with the perinatal damages of CNS by the account of changes LPO in red corpuscles.

Methods: An analysis was conducted for 70 pectoral children of age with the perinatal damages of CNS and with pathogeny of consequences of perinatal damages of the nervous system. Oxibral rendered the positive operating on the maintenance of MDA after incubation. At comparing of the indicated index it is not reduced to the norm of statistical difference.

Results: The correction of "oxibral" of neurological violations opens the prospect of rehabilitation and assists a considerable reduction of per cent of children with the remaining phenomena of perinatal damages of CNS. Peroxide oxidation of lipids indicators in healthy infants have their characteristics, and the studies carried out can be used as controls for various pathological conditions, including perinatal CNS injuries.

Conclusions: Thus, it was found that LPO indicators in healthy infants have their characteristics, and the results of the studies carried out can be used as controls for various pathological conditions, including perinatal CNS injuries.

Key Words: Peroxide oxidation of lipids, Malonic dialdehyde, Degree of hemolysis of red corpuscles, The symptom of Grefe, Oxibral, Damages of CNS

\section{INTRODUCTION}

The perinatal damages of the brain make more than 60 per cent pathology of the nervous system of child's age, directly participate in the development of such diseases, as child's cerebral paralysis, epilepsy, minimum cerebral disfunction, that, in turn, assist the increase of recurrent respiratory diseases Presently the basic hypothesis of a pathogeny of consequences of perinatal damages of the nervous system (CPDNS) is cerebrovascular violations. In the conditions of hypoxia peroxide oxidization of lipids is violated with the accumulation of aggressive free radicals, hydroperoxides that render the destructive operating on the membranes of neurons. ${ }^{1}$

In case of impaired cerebral circulation in newborns, several researchers conclude about the intensity of lipid peroxida- tion in this pathology based on an increase in the content of LPO products. Only with an integrated approach, i.e. using several research methods in combination, one can count on the possibility of a more complete assessment of pathological changes in plasma membranes. ${ }^{2}$

As you know, LPO indicators characterize not only the severity of the pathological process but to a certain extent the effectiveness of the correction of disturbed metabolism. Consequently, according to these indicators, it is possible not only to assess the degree of pathological changes but also, most importantly, the effectiveness of the therapy. ${ }^{3}$

Recently, several experimental and clinical studies have appeared, indicating the beneficial effect of the new herbal preparation oxybral on circulatory and metabolic cerebral disorders. The active ingredient of oxybral is a vinca alkaloid

\section{Corresponding Author:}

Sharipov R. Kh., Samarkand State Medical Institute, PhD., Teacher of the Department of Pediatrics FADE, Samarkand, Uzbekistan.

ISSN: 2231-2196 (Print)

Received: 30.09 .2020
ISSN: 0975-5241 (Online)

Revised: 24.10 .2020
Accepted: 28.11 .2020

Published: 03.02 .2021 
vincamine. Numerous studies have confirmed the presence of an active metabolic effect of oxybral on ischemic tissue. The drug helps to increase the consumption and use of oxygen and glucose by brain tissues improves and normalizes glycolysis, is well tolerated by patients. It has been proven that the drug is rapidly absorbed for any method of application, uniformly distributed in the brain tissues, metabolized and eliminated through the kidneys. A great deal of clinical experience has been accumulated confirming the selective vaso regulatory effect of vincamine on cerebral vessels. ${ }^{3,4}$

However, there are no studies in which the effect of this drug in perinatal damage to the nervous system in children of the first year of life would be studied, the questions of the optimal dosage and duration of the course of using this drug are not substantiated. ${ }^{5,6}$

The work aimed to substantiate the therapy with oxybral in infants with perinatal CNS injuries by taking into account changes in the processes of lipid peroxidation (LPO) in erythrocytes. We were the first to study the effect of oxybral on the state of the nervous system in CPDNS based on studying LPO processes

\section{MATERIALS AND METHODS}

The paper analyzes the results of clinical and biochemical studies in 70 children of the first year of life. The main group consisted of 44 infants with perinatal CNS injuries. The control group included 26 children with CPDNS who have not prescribed the drug we tested. Since the processes of lipid peroxidation have age-related dynamics, we also studied the state of LPO processes in 20 healthy children.

The state of lipid peroxidation in erythrocytes was assessed according to the following indicators: the degree of erythrocyte hemolysis before incubation (mechanical stability of erythrocytes) and after incubation under physiological conditions (peroxide hemolysis without incubation), the MDA content in erythrocytes, the MDA coefficient/hemolysis after incubation of MDA in the intensity of erythrocyte degradation. To determine lipid peroxidation parameters, only 170 $\mu \mathrm{l}$ of erythrocytes (approximately $0.7-0.8 \mathrm{ml}$ of blood) were required. Determination of the activity of LPO processes in erythrocytes was studied twice: before and after treatment. ${ }^{7,8}$

\section{RESULTS}

Most children with perinatal CNS damage were born to mothers suffering from chronic diseases of the cardiovascular system, endocrine system, nasopharynx, kidneys, digestive organs, and genitals. Complicated pregnancy was observed in all mothers of children with CPDNS. All children were born to mothers with a complicated course of labour.
In the neurological status, the hypertensive-hydrocephalic syndrome was $42.8 \%$ and was characterized by the opening of the sagittal suture more than $0.5 \mathrm{~cm}$, the large fontanelle over $3 \times 3 \mathrm{~cm}$, non-closure of the small fontanelle in most children, pronounced development of collateral veins of the head, and baldness of the head. Various ocular symptoms were found in the form of Graefe's symptom, "setting sun", and unstable horizontal nystagmus. The diagnosis was confirmed by ECHOEG, and upon examination of the fundus, by the detection of retinal vasospasm.

The main changes in the syndrome of vegetative-visceral dysfunctions $(38.5 \%)$ were persistent regurgitation, persistent hypotrophy, respiratory rhythm disturbances, skin colouration, acrocyanosis, paroxysms of tachy, bradypnea, thermoregulation disorders, gastrointestinal dysfunction, temporal lobe hair loss.

With the syndrome of increased neuro-reflex excitability (11.4\%), against the background of normal mental and physiological development, the child reveals emotional lability, motor anxiety arising from minor changes in the environment, when exposed to visual, auditory, tactile analyzers. Such children have a periodic small-amplitude tremor, startle, difficulty falling asleep, anxious superficial, not long enough sleep.

Psychomotor development retardation syndrome (7.1\%) is characterized by impaired reduction of unconditioned congenital reflexes. Children are not actively interested in toys and surrounding objects, do not react enough to the presence of their mother, humming is inactive and short-lived, manipulations with objects are delayed, there is no active attention.

In the department, children of the control group received complex therapy: drugs that improve cerebral circulation (vinpocetine), piracetam was used, and syndrome therapy was performed. Depending on which clinical manifestations prevailed, they used anticonvulsants, diuretics and vitamin preparations. Parallel correction of disorders caused by underlying diseases was also carried out.

\section{DISCUSSION}

The results of the research showed that children with perinatal CNS injuries were found to have significant disorders in LPO processes, which can be characterized as a decrease, and in some cases, their imbalance. This was evidenced by a significant increase in the MDA content before and after incubation, the MDA / hemolysis ratio after incubation, an increase in erythrocyte hemolysis after incubation, and a decrease in the percentage of increase in hemolysis compared to the data. ${ }^{5,9,10}$

In children of the control group, the hemolysis of erythrocytes before incubation, although significantly reduced com- 
pared to the data before treatment, still does not normalize. Hemolysis of erythrocytes after incubation practically did not differ from those of healthy children. Therefore, despite the increased value of erythrocyte hemolysis before incubation, the hemolysis of erythrocytes after incubation in children of this group does not differ from those of healthy children. ${ }^{6,11,12}$ In this regard, the percentage of increase in erythrocyte hemolysis in children of this group was significantly reduced in comparison with healthy children. Before incubation, the MDA content remained at high values, although there was a tendency to decrease it. In children of this group, MDA after incubation was significantly higher than the norm, however, the treatment carried out contributed to a significant decrease in this indicator compared with the data before treatment. ${ }^{13-14}$

The MDA / hemolysis ratio after incubation was higher than in healthy children, but there was a significant decrease in comparison with the initial data. The intensity of MDA degradation significantly increased in comparison with the initial data and did not differ from the indicators of children in the healthy group. The same changes were observed in the degradation / MDA ratio. ${ }^{7,8}$

Thus, in children with AED who received conventional therapy, there is a significant positive dynamics in the indicators of LPO processes, but most of them do not reach the norm. Positive dynamics was also noted in terms of clinical symptoms: children became calmer, their sleep returned to normal, the size of the brain ventricles gradually normalized, and the functions of internal organs stabilized. Appetite improved and, therefore, the children began to gain weight. An active interest in the environment appeared. In general, clinically significant improvement was observed between 10-15 days of therapy. ${ }^{15}$

The presence of changes in LPO processes in children with perinatal CNS injuries, which persist despite the course of therapy, dictates the need to include new drugs in the treatment complex, the action of which is more effective. We have used a new herbal preparation oxybral, the effect of which in infants has been insufficiently studied. High efficiency, absence of side effects, as well as good tolerance, served as the basis for the appointment of Oxybral for the correction of cerebral and metabolic disorders and, indirectly, for the correction of LPO in children with perinatal CNS injuries. ${ }^{3,4}$

Given the fact that infants were prescribed oxybral for the first time, it became necessary to scientifically substantiate the appointment of this drug, to select the dose and duration of the course of treatment based on studying the effect on LPO in erythrocytes. To determine the dose and duration of the course of treatment, initially, oxybral was prescribed at $7.5 \mathrm{mg}$ /day. Complete normalization of indicators in most children was observed between 7 and 10 days.
Treatment with oxybral turned out to be most effective when applied for 10 days. For an objective assessment of the therapeutic effect of oxybral, the results of treatment of these patients were compared with the corresponding data in children of the control group. Erythrocyte hemolysis before incubation in children of the study group did not differ from the data obtained in healthy children and was significantly lower than in the control $(1.4 \pm 0.05 \%$ and $1.17 \pm 0.12 \%$, respectively). When comparing the hemolysis of erythrocytes after incubation in children with AED who received oxybral with the data obtained in healthy children and children of the control group, no statistically significant difference was found $(2.37$ $\pm 0.16 \%, 2.21 \pm 0.44 \%$ and $2,3 \pm 0.03 \%$, respectively). Due to the normalization of mechanical and peroxide hemolysis, the percentage of increase in hemolysis did not differ from the data of healthy children and was significantly higher than in the control group (99.7\% and 64\%, respectively).

The MDA content before incubation in children of the main group did not differ from the data obtained in the control study. That is, despite the course of therapy with oxybral, the MDA content before incubation in children with PEP remained significantly higher than in healthy children $\left(2.7 \pm 0.24 \mathrm{nmol} / 10^{6}\right.$ erythrocytes versus $0.86 \pm 0.12 \mathrm{nmol} / 10^{6}$ erythrocytes). However, one cannot fail to note a significant decrease in this indicator compared with the initial data $\left(2.7 \pm 0.24 \mathrm{nmol} / 10^{6}\right.$ erythrocytes versus $3.3 \pm 0.33 \mathrm{nmol} / 10^{6}$ erythrocytes).

Oxybral had a positive effect on the MDA content after incubation. When comparing this indicator with the norm, no statistical difference was found $\left(1.6 \pm 0.18 \mathrm{nmol} / 10^{6}\right.$ erythrocytes and $1.4 \pm 0.16 \mathrm{nmol} / 10^{6}$ erythrocytes). At the same time, in the children of the main group, the MDA content after incubation was significantly lower than in the control $\left(1.6 \pm 0.18 \mathrm{nmol} / 10^{6}\right.$ erythrocytes and $2.0 \pm 0.17 \mathrm{nmol} / 10^{6}$ erythrocytes, respectively ). $., 9,10$

The MDA / hemolysis ratio after incubation when receiving oxybral did not differ from the data in the control group and was significantly higher than normal values $(0.9 \pm 0.2$, $1.0 \pm 0.07$ and $0.5 \pm 0.1$, respectively). Clinical symptoms also had a pronounced positive trend: children became calm and active. Sleep has returned to normal. Normalization of ECHOEG parameters against the background of the use of oxybral was noted much faster (7-10 days, versus 10-15 in the control, depending on the degree of the initial change). Pulse and breathing became rhythmic, the activity of the gastrointestinal tract stabilized, the children began to gain weight. The temperature decreased or returned to normal, regardless of the use of antipyretic drugs in children with a history of unmotivated febrile conditions. Children began to take an active interest in their surroundings, and a tendency to develop motor skills began to appear. In general, a pronounced clinical effect was observed on days 6-10 of therapy (Table 1). 
Table 1: Contain of base groups

\begin{tabular}{|c|c|c|c|}
\hline & Base group & $\begin{array}{l}\text { Control } \\
\text { group }\end{array}$ & $\begin{array}{l}\text { Health chil- } \\
\text { dren }\end{array}$ \\
\hline $\begin{array}{l}\text { The MDA content } \\
\text { before incubation }\end{array}$ & $\begin{array}{l}1.6 \pm 0.18 \\
\mathrm{nmol} / 10^{6}\end{array}$ & $\begin{array}{l}2.0 \pm .0 .17 \\
\mathrm{nmol} / 10^{6}\end{array}$ & $\begin{array}{l}1.4 \pm 0.16 \\
\mathrm{nmol} / 10^{6}\end{array}$ \\
\hline $\begin{array}{l}\text { The MDA content } \\
\text { after incubation }\end{array}$ & $\begin{array}{l}2.7 \pm 0.24 \\
\mathrm{nmol} / 10^{6}\end{array}$ & $\begin{array}{l}2.7 \pm 0.24 \\
\text { нмоль } / 10^{6}\end{array}$ & $\begin{array}{l}0.86 \pm 0.12 \\
\text { nmolio }^{6}\end{array}$ \\
\hline $\begin{array}{l}\text { The MDA content } \\
\text { before incuba- } \\
\text { tionthe initial } \\
\text { data }\end{array}$ & $\begin{array}{l}3.3 \pm 0.33 \\
\mathrm{nmol} / 10^{6}\end{array}$ & $\begin{array}{l}2.7 \pm 0.24 \\
\mathrm{nmol} / 10^{6}\end{array}$ & $\begin{array}{l}0.86 \pm 0.12 \\
\mathrm{nmol} / 10^{6}\end{array}$ \\
\hline $\begin{array}{l}\text { Erythrocyte } \\
\text { hemolysis before } \\
\text { incubation }\end{array}$ & $1.4 \pm 0.05 \%$ & $1.170 .12 \%$ & $1.4 \pm 0.05 \%$ \\
\hline $\begin{array}{l}\text { Erythrocyte } \\
\text { hemolysis after } \\
\text { incubation }\end{array}$ & $2.37 \pm 0.16 \%$ & $2.21 \pm 0.44 \%$ & $2.3 \pm 0.03 \%$ \\
\hline $\begin{array}{l}\text { The MDA / he- } \\
\text { molysis ratio after } \\
\text { incubation }\end{array}$ & $0.9 \pm 0.2$ & $1.0 \pm 0.07$ & $0.5 \pm 0.1$ \\
\hline
\end{tabular}

\section{CONCLUSION}

Thus, it was found that LPO indicators in healthy infants have their own characteristics, and the results of the studies carried out can be used as controls for various pathological conditions, including perinatal CNS injuries. A scientifically substantiated approach to the appointment of oxybral for the correction of perinatal CNS injuries under the control of changes in LPO processes was carried out. Studies have shown that the appointment of children with perinatal damage to the central nervous system oxybral. Along with the normalization of most indicators, LPO contributes to more rapid normalization of neurological symptoms. The possibility of correcting neurological disorders with oxybral opens up the prospect of rehabilitation and contributes to a significant reduction in the percentage of children with residual symptoms of perinatal CNS damage.

\section{ACKNOWLEDGMENT}

Authors acknowledge the immense help received from the scholars whose articles are cited and included in references to this manuscript. The authors are also grateful to authors / editors / publishers of all those articles, journals, and books from which the literature for this article has been reviewed and discussed.

\section{Conflict of Interest: Nil}

Source of Funding: Nil

\section{REFERENCES}

1. Barashnev YI. Hypoxic encephalopathy: hypotheses of the pathogenesis of cerebral disorders and the search for methods of drug therapy. Russian Bull Perinatol Pediatr 2002;5(6):45-49.

2. Volodin NN, Medvedev MI, Rogatkin SO. Perinatal encephalopathy and its consequences - controversial issues of semiotics and therapy. Russian Pediatr J 2001;12(4):56-61.

3. Akhmedov FK. Features of renal function and some indicators of homeostasis in women with mild preeclampsia. Eur Sci Rev (Austria) 2015;4(5):58-60.

4. Akhmedov FK, Avakov VE, Negmatullaeva MN. Status of cardio-hemodynamics and cardiac geomerms in women with complications with severe preeclampsia. News Dermatol Reprod Health 2017;3(I):27-29.

5. Guryeva MV, Yu. B, Kotov VA. Daily monitoring of blood pressure and heart rate in the diagnosis. Russian Bull Obstet Gynecol 2013;3:4-9.

6. Garg SA, Chakravarti R, Singh NR, Masthi RC, Goyal GR, Jammy E, Dengue serotype-specific Seroprevalence among 5to 10 -Year-Old Children in India: A Community-Based CrossSectional Study. Int J Infect Dis 2017;54:25-30.

7. Taksande A, Meshram R, Lohakare A. A Rare Presentation of Isolated Oculomotor Nerve Palsy Due to Multiple Sclerosis in a Child. Int J Pediatr 2017;5(8):5525-5529.

8. Prusakov VF, Morozov EA, Marulina VI, Belousova MV, Utkuzova MA, Gamirova RG, Knyazeva OV. The role of perinatal damage to the nervous system in the formation of neurological pathology in childhood.Vestnik modern Clin.Med. 2016; 9(2): 65-70.

9. Vasiliev V, Tyagunova AV, Drozheva VV. Renal function and indicators of

10. Wu YW, Colford Jr JM. Chorioamnionitis as a risk factor for cerebral palsy: a meta-analysis. Jama. 2000 Sep 20;284(11):141724.

11. Mallick S. Study On The Clinical Profile Of Patients With Cerebral Palsy (Doctoral dissertation) 2011.

12. Puri S, Fernandez SA. Puranik D. Anand, A. Gaidhane Z. Quazi S, Patel S. Policy Content and Stakeholder Network Analysis for Infant and Young Child Feeding in India. BMC Public Health. 2017; 17:217-221.

13. Savchenkov Y, Soldatova O, Shilov S. Age physiology (physiological characteristics of children and adolescents).Textbook for universities 2019:182.

14. Dautov AA. HPLC determination of malonic dialdehyde in plasma and saliva. Sorption Chromatogr Proc 2018;18(1):73-82.

15. Williams CE, Mallard EC, Tan WKM, Gluckman PD. Pathophysiology of perinatal asphyxia. Clin Perinatol 1993;3(4):9194 . 